\title{
A DIABETOLOGIA SOCIAL EM PORTUGAL. GÉNESE, INFLUÊNCIAS E CONCRETIZAÇÕES (1926-1931)
}

\author{
Ismael Cerqueira Vieira \\ Universidade de Coimbra \\ Email: ivieira@uc.pt \\ ORCID iD: https://orcid.org/0000-0003-2054-5123 \\ João Rui Pita \\ Universidade de Coimbra \\ Email: jrpita@ci.uc.pt \\ ORCID iD: https://orcid.org/0000-0003-2851-337X

\section{Ana Leonor Pereira} \\ Universidade de Coimbra \\ Email: aleop@ci.uc.pt \\ ORCID iD: https://orcid.org/0000-0003-3581-1359
}

Recibido: 2 junio 2020; Aceptado: 28 febrero 2021

Cómo citar este artículo/Citation: Cerqueira Vieira, Ismael; Rui Pita, João; Pereira, Ana Leonor (2021) “A diabetologia social em Portugal. Génese, influências e concretizações (1926-1931)", Asclepio, 73 (2): p567. https://doi.org/10.3989/asclepio.2021.25

RESUMO: A diabetes é uma epidemia silenciosa e debilitadora que tem recebido uma atenção redobrada nas últimas décadas por parte da Medicina. Este trabalho pretende realizar uma análise sobre o aparecimento da diabetologia social em Portugal, centrado no segundo quartel do século XX. Primeiramente pretende-se perceber a génese desta área médica, tendo por base o trabalho desenvolvido pelo médico Ernesto Roma em Lisboa. Segue-se uma análise focada no rastreamento das correntes de pensamento médico que influenciaram a diabetologia portuguesa e por último pretende-se perceber como foi organizado o modelo de assistência aos diabéticos nos primeiros tempos da sua existência.

PALAVRAS-CHAVE: Diabetologia Social; Ernesto Roma; Portugal; Associação Portuguesa dos Diabéticos Pobres-APDP.

\section{SOCIAL DIABETOLOGY IN PORTUGAL. GENESIS, INFLUENCES AND ACHIEVEMENTS (1926-1931)}

\begin{abstract}
Diabetes is a silent and debilitating epidemic that has received increased attention in recent decades by Medicine. This work intends to carry out an analysis of the emergence of social diabetology in Portugal, centered in the second quarter of the 20th century. Firstly, it is intended to understand the genesis of this medical area, based on the work developed by the physician Ernesto Roma in Lisbon. This is followed by an analysis focused on tracking the currents of medical thought that influenced Portuguese diabetology and, finally, it is intended to understand how the model of assistance to diabetics was organized in the early days of its existence.
\end{abstract}

KEY WORDS: Social Diabetology; Ernesto Roma; Portugal; Associação Portuguesa dos Diabéticos Pobres-APDP. 


\section{INTRODUÇÃO}

A diabetes é uma doença crónica que atualmente está no top 10 das causas de morte em todo o planeta. Por ser uma ameaça à saúde global a Organização Mundial de Saúde tem como meta a redução de $30 \%$ de mortes prematuras devidas a esta doença até 2030 . Contudo, as preocupações médicas com esta doença afirmaram-se sobretudo nos inícios do século $\mathrm{XX}$, até porque anteriormente a esse período a diabetes era uma doença pouco frequente. Com as alterações dos padrões de consumo alimentar nos países industrializados a diabetes, tipo 2 ou insulino-resistente, passou a ter maior destaque na epidemiologia de vários países.

Nos Estados Unidos da América surgiu a diabetologia social ${ }^{1}$ no primeiro quartel do século $X X$, com importantes avanços ao nível da terapêutica, prevenção e educação dos doentes. Numa primeira fase, até 1921-1922, fizeram-se importantes avanços no campo da dietoterapia. Estudos e experiências desenvolvidas na época permitiram conceber o primeiro tratamento lógico para a redução da glicosúria através da restrição alimentar. Após a descoberta da insulina o tratamento geral da diabetes foi redefinido, dando-se maior autonomia aos doentes, o que implicou desenhar planos educativos que permitissem o seu empoderamento.

No centro dessa revolução terapêutica e do nascimento da diabetologia social em Boston esteve um médico português. Inicialmente visando fazer estudos de pós-graduação em Ética na Universidade de Harvard, Ernesto Roma assistiu em 1922 e 1923 à emergência desta área médica, tendo, absorvido os conhecimentos e modernos modelos de assistência.

Em 1923, Ernesto Roma regressou a Portugal na posse de conhecimentos e métodos de tratamento modernos, dedicando-se a estabelecer as bases da diabetologia social em Portugal. A ideia de fundar uma associação para assistir os diabéticos pobres tornou-se possível em 1926, criando na Associação Protetora dos Diabéticos Pobres (APDP) uma verdadeira escola de diabetes que rapidamente se estendeu ao resto do país.

Deve referir-se que são escassos os estudos sobre a história da diabetes em Portugal e sobre a própria receção da insulina. Sobre a diabetologia e a história da endocrinologia em Portugal refiram-se os estudos de Ismael Vieira A Diabetologia em Portugal: Contributos para a sua História (2020), bem como História da Endocrinologia em Portugal no Século XX (2018). Entre os estudos afins e com interesse para a compreensão do tema refiram-se os estudos de António Barros Veloso e Luís Gardete Correia sobre a Associação dos Diabéticos Pobres, Ernesto Roma e a Associação dos Diabéticos Pobres (2017). Com menor dimensão citem-se os estudos de Luís da Silveira Botelho, Contribuição para a história da endocrinologia em Portugal (1988) e o estudo generalista e já muito datado de Augusto Celestino da Costa, Comentários sobre o advento e a evolução da endocrinologia (1952). Ao nível internacional esta área de estudo tem também colhido bastante interesse².

Este artigo centra-se na análise da génese da diabetologia social em Portugal como área médica e social com base no trabalho realizado pelo médico Ernesto Roma, na primeira fase entre 1926 e 1931 . Neste sentido pretende-se perceber quais as correntes de pensamento médico que o influenciaram, bem como se organizou a assistência e a educação aos diabéticos pobres no período temporal tratado.

\section{ANTECEDENTE E gÉnESE DA QuEStÃo DIABETOLÓGICA EM PORTUGAL}

No início do século XX, em Portugal, era pouca a informação que circulava acerca da diabetes e da sua terapêutica. Nessa época, a diabetes era uma doença que passava despercebida nas estatísticas obituárias oficiais, pelo escasso número de óbitos existentes (pela raridade da doença ou pelas dificuldades de diagnóstico da época), de tal modo que somente a partir de 1931 a doença passou a figurar no "Anuário Estatístico de Portugal» (Cerqueira Vieira, 2020, p. 146). Se recuarmos até ao século XIX, para fazer um ponto da situação, percebemos que a bibliografia existente era escassa.

Em 1806, Manoel Pereira da Graça, Doutor em Medicina pela Universidade de Coimbra, escreveu um "Tratado da Diabetes», onde pretendia fazer um "estado da arte" sobre a diabetologia do seu tempo, denunciando as teorias erradas perfilhadas pelos médicos seus contemporâneos e concluindo que "a Diabetes é uã daquelas [doenças], sobre o qual os nossos conhecimentos estão ainda tão atrasados, que se ignora a sua natureza, e curativo" (Graça, 1806, p. IV).

Abel Jordão, doutorado pela Universidade de Paris e professor da Escola Médico-cirúrgica de Lisboa, foi outro médico que se dedicou ao estudo da diabetes. Na sua tese doutoral "Considérations sur un cas de diabète» (Jordão, 1857), fazia menção sistemática à sintomatologia da doença e à raridade de casos observados. Quanto à terapêutica, Jordão concebia tratamentos com várias substâncias farmacológicas que incluíam água de Vichy, limonada nítrica com éter, 
fricções narcóticas nos rins, pilulas purgativas, banhos de vapor e medicamentos sudoríficos, que deviam ser acompanhados de uma dieta que incluía alimentos feculentos, vegetais ou pão de glúten por exemplo (Jordão, 1857, p. 76-80). Publicou anos mais tarde outros estudos sobre a doença empenhando-se em aprofundar o conhecimento sobre as causas da diabetes (Jordão, 1863a; Jordão, 1863b) e a sua sintomatologia (Jordão, 1866).

As teses de final de curso médico-cirúrgico, ditas teses inaugurais das antigas Escolas Médico-cirúrgicas de Lisboa e Porto, constituem geralmente fontes dignas de estudo. Não obstante, no campo da diabetologia pouco contribuíram para o avanço científico no período anterior ao século $\mathrm{XX}$, limitando-se a replicar os conhecimentos em voga na época ${ }^{3}$.

Nos inícios do século $X X$, anteriormente à descoberta da insulina, deve mencionar-se dois estudos dignos de nota. O primeiro trata-se da tese doutoral de Elísio Coimbra realizada na Faculdade de Medicina do Porto, com base em casos observados no Hospital Geral de Santo António, e cuja principal conclusão foi de que a diabetes "A diabete não tem um tratamento específico" (Coimbra, 1919, p. 139). O segundo estudo, de José de Sousa Costa, tinha como objetivo o estudo do tratamento da diabetes pelo método da restrição alimentar de Allen, baseado no controlo da glicosúria pela subnutrição através do jejum (Costa, 1922).

Nos inícios do século $X X$, a comunidade científica internacional sabia que o pâncreas era a sede da doença e que as ilhotas de Langerhans eram as responsáveis pela segregação da insulina, uma hormona que permitia o metabolismo do açúcar. Apesar dos vários aportes e pistas que a ciência médica da primeira metade do século XIX veio dar ao problema da diabetes, a doença continuava a ser um mistério. Foi preciso chegar a meados do século XIX para que uma área do saber médico em expansão, a fisiologia, começasse a dar os primeiros frutos para se aclarar determinados aspetos desta patologia. Em 1848, Claude Bernard realizou um conjunto de descobertas sensacionais, percebendo que o suco pancreático tinha uma função digestiva e que o fígado tinha uma função glicogénica (Holmes, 1999, pp. 75-76). Em Berlim, Rudolf Virchow (18211902) e Wilhelm Kühne (1837-1900) reavivaram o interesse pela descoberta do pâncreas isolando enzimas pancreáticas e Paul Langerhans fez a primeira descrição da estrutura microscópica de um pâncreas normal, descobrindo as ilhotas pancreáticas. Oscar Minkowski (1858-1931) e Joseph von Mering (1849-1908) descobriram que a extração total do pâncreas num cão levou o animal a desenvolver diabetes açucarada, demonstrando assim que a diabetes ocorria na ausência ou extirpação do pâncreas (Houssay, 1989, p. 350).

Nos finais do século XIX alguns médicos preconizavam a opoterapia com extratos de pâncreas, como Edouard Laguesse, Emmanuel Hédon, George Zuelzer ou ainda Israel Kleiner (Cerqueira Vieira, 2020, pp. 3437), buscando uma substância capaz de permitir o controlo da glicemia. Os sucessivos fracassos na descoberta da substância que controlaria o açúcar no sangue, o que viria só a efetivar-se entre 1921-1922, fez com que a tónica do tratamento da diabetes fosse posta na dieta alimentar. Embora os tratamentos alimentares baixos em hidratos de carbono e o jejum já fossem conhecidos desde o século XVIII, foram os estudos científicos de Frederick Allen, Edgar Stillman e Reginald Fitz ${ }^{4}$ e ainda de Elliot Joslin, o mais famoso diabetologista norte-americano do século $\mathrm{XX}$, que fizeram dele o primeiro modelo racional de tratamento da diabetes.

Foi neste contexto que Ernesto Roma, um jovem médico português e veterano da Grande Guerra, teve um contacto internacional com a diabetologia social norte-americana, o que viria a mudar a sua inclinação inicial para a psiquiatria e neurociências. Após ter terminado o curso de Medicina na Escola Médico-cirúrgica de Lisboa/Faculdade de Medicina em 1913, com a tese "Microcefalia», iniciou carreira médica no Hospital Escolar de Santa Marta em Lisboa onde exerceu funções de assistente de Anatomia e segundo assistente de Patologia Interna, Clínica Médica, Terapêutica e Especialidades Médicas, trabalhando com Francisco Pulido Valente e, sobretudo, com Carlos Bello de Moraes, que o viria a aconselhar a fazer especialização em Ética no Estados Unidos da América (EUA) com Richard Clarke Cabot (Veloso \& Correia, 2017, p. 246-247).

Cabot criou e liderou o primeiro departamento hospitalar de serviço social nos EUA (Garchar \& Kaag, 2013, p. 553) e após o seu regresso de França, onde combateu durante a Grande Guerra, foi-lhe oferecida, em 1920, a presidência do Departamento de Ética Social na Universidade de Harvard, departamento esse que oferecia cursos de pós-graduação destinados a preparar os médicos para o campo específico do serviço social (O’Brien, 1985, p. 546).

A génese da diabetologia social em Portugal esteve ligada, nos seus inícios, às atividades clínica e educativa do médico Ernesto Roma. Em 1922, este médico rumou a Boston, nos EUA, para estagiar no Bent Brigham Hospital e no Massachusetts General Hospital e especializar-se em Ética, mas o que aprendeu e viu nos EUA permitiu-Ihe reorganizar a sua vida pessoal e 
profissional e regressar a Portugal para iniciar a área da diabetologia social ${ }^{5}$.

No essencial, Ernesto Roma teve contacto com a área emergente do Serviço Social, cujo principal mentor foi Richard Cabot, o supervisor de Roma, e com a primeira escola de diabetologia no mundo iniciada por Elliott Joslin - a Joslin Clinic em Boston - onde aprendeu as bases para o tratamento da diabetes, designadamente a terapêutica alimentar específica, a educação/formação dos doentes e dos profissionais de saúde envolvidos na assistência e a insulinoterapia, que dava os primeiros, e decisivos, passos.

O regresso de Ernesto Roma a Portugal traduziu-se no despontar da diabetologia clínica e social. No Hospital de Santa Marta e na clínica privada pôs em prática as bases do tratamento e educação dos diabéticos que aprendera em Boston e foi mais além ao criar a Associação Portuguesa dos Diabéticos Pobres (APDP) em 1926, com a qual iniciou a diabetologia social. Mais do que uma associação destinada a assistir os diabéticos carenciados revelou-se um espaço de investigação sobre a doença e de formação dos profissionais de saúde portugueses dedicados à diabetes mellitus.

\section{INFLUÊNCIAS DA DIABETOLOGIA NORTE-AME- RICANA EM PORTUGAL}

A diabetologia como área médica dedicada ao estudo, tratamento e prevenção da diabetes remonta aos finais do século XIX e inícios do século XX. O aparecimento e diferenciação de áreas médicas em especialidades nos finais do século XIX esteve ligado não só ao volume do saber médico acumulado como à estrutura social onde a Medicina era praticada. Por um lado, um conhecimento médico mais profuso, científico e sistemático e, por outro, novos modelos de viver e de trabalhar nas sociedades contemporâneas potenciaram o aparecimento de especialidades e subespecialidades que permitiam estudar, tratar, assistir e prevenir as doenças próprias das sociedades ocidentais ou ocidentalizadas.

Queremos com isto dizer que nos inícios do século XX houve modificações importantes na morbilidade ocidental que, segundo Lain-Entralgo (1978, p. 643), ficaram patentes em quatro grandes grupos - as doenças infeciosas, as sociais, as neoplásicas e as metabólicas. À medida que as doenças infeciosas foram sendo progressivamente dominadas e debeladas, com os progressos sanitários, a melhoria da alimentação e habitação das populações e a vacinação preventiva, pese embora o surgimento de epidemias graves como a gripe pneumónica de $1918 / 1919^{6}$, foram aumentando exponencialmente as doenças oncológicas, devido aos novos estilos de vida e ao aumento da esperança média de vida. Também as doenças sociais, nomeadamente as de desgaste, as devidas ao trabalho, as de má nutrição e as doenças psicóticas como a depressão, o transtorno por défice de atenção e hiperatividade, as perturbações da personalidade e a síndrome de burnout ${ }^{7}$ ganharam expressão. As doenças metabólicas completam o quadro da morbilidade contemporânea, com doenças cada vez mais difusas entre a população, de etiologia tanto genética como ambiental, como a diabetes (Lain-Entralgo, 1978, p. 645).

\subsection{NOVAS (E VELHAS) TERAPÊUticas}

$O$ advento e o desenvolvimento da diabetologia enquadram-se neste panorama mais alargado, tanto de desenvolvimento das ciências endocrinológicas e da nutrição como ainda nas transformações socioeconómicas das sociedades ocidentais nos inícios do século $\mathrm{XX}$. Superadas as questões clínicas principais ao nível da anatomopatologia e a fisiopatologia da diabetes, o assunto premente da Medicina era o tratamento da diabetes, o que veio a originar duas escolas distintas.

De um ponto de vista mais conservador encontravam-se os médicos que defendiam que a insulina era um novo meio terapêutico, o que não invalidava as aprendizagens anteriores como a dietoterapia específica, baseada numa restrição calórica, e os preceitos higiénicos aplicados aos diabéticos. De um ponto de vista mais liberal estavam os que acreditavam que a diabetes era uma patologia devida à carência de insulina, consequentemente os doentes podiam comer tudo sem restrições, bastando apenas acertar com a dosagem precisa de insulina, o que implicava fazer tábua rasa dos conhecimentos e das experiências terapêuticas anteriores (Lisboa, 2002, pp. 12-13).

A descoberta da insulina em Toronto, por Frederick Banting e Charles Best em 1921-1922, constituiu um marco decisivo na terapêutica da diabetes, estando esta descoberta a par da descoberta da penicilina e da estreptomicina, considerada por muitos como medicamentos miraculosos (Bynum, 2012, p. 228). A descoberta da insulina representou um progresso assinalável no tratamento da diabetes mellitus, sendo a hormona capaz de resgatar os doentes do coma e de uma morte certa (Bynum, 2012, p. 228).

A orientação mais conservadora estava representada por médicos como Frederick Allen, dos quais Ernesto Roma colheu alguns métodos de trabalho e modalidades dietoterapêuticas. Os tratamentos dieté- 
ticos racionais da diabetes eram usados desde o século XVIII, por médicos como John Rollo, John Camplin ou Frederick Pavy, baseados em dietas hipocalóricas e no jejum, como forma de controlar a glicosúria dos doentes (Tattersall, 2009). Mas, o expoente máximo da dietoterapia da diabetes surgiu nos inícios do século XX com os estudos de Frederick Allen. O primeiro grande estudo - «Studies Concerning Glycosuria and Diabetes» (1913) -, com mais de mil páginas, resultou de uma extensa investigação realizada na Harvard Medical School entre 1909 e 1912, onde defendia uma dieta restritiva caracterizada pela baixa quantidade de hidratos de carbono - a que chamou "dieta dos esquimós" - que pressupunha diminuir a quantidade de comida ingerida até a urina ficar isenta de açúcar (Tattersall, 2009, pp. 46-47).

Noutro estudo copublicado em 1919 - «Total Dietary Regulation in the Treatment of Diabetes» - enquanto investigador do Rockefeller Institute for Medical Research, aprofundou um tipo de tratamento que, para além de incluir uma dietoterapia doseada ao miligrama, incluía igualmente planos de dieta até e após a cessação da glicosúria, o exercício físico como forma de controlo dos hidratos de carbono, e cuidados rotineiros dos doentes, com especial enfase em evitar o confinamento na cama, o vestuário, os banhos, a catarse, a medicação acessória e a gestão de complicações advindas do estado diabético (Allen, Stillman \& Fitz, 1919).

A partir da descoberta da insulina surgiu uma orientação mais liberal encabeçada por Frederick Banting, que veiculava a ideia de que os diabéticos podiam comer de tudo, já que era apenas necessário acertar na dose correta de insulina. Esta visão foi bem acolhida, sobretudo, pelos pediatras que podiam retirar as crianças diabéticas do estado de subnutrição em que se encontravam (Lisboa, 2002, p. 12). Todavia, foi uma visão integrada e holística da condição diabética que vingou com a inclusão da educação do diabético, que pretendia pôr os doentes no centro dos cuidados médicos, e o seu empoderamento como agente principal de transformação da sua nova condição de saúde.

\subsection{EDUCAÇÃO DOS DIABÉTICOS}

A descoberta e a aplicação da insulina no tratamento da diabetes na década de 1920 veio provar a cronicidade da doença, implicando que a insulinoterapia estivesse na interceção com outros preceitos terapêuticos como a dieta, o exercício físico, os cuidados com as infeções, o protocolo medicamentoso, entre outros (Gardner, 2019, p. 177). A diabetes era uma doença crónica, associável a outras complicações infeciosas, vasculares, oftálmicas, necróticas, etc., o que exigia um tratamento ao longo da vida do doente e o conhecimento específico da doença, o que fez com que a diabetologia penetrasse no lar dos doentes, levando a uma domesticação dos conhecimentos científicos e das tecnologias médicas implicadas.

Nesta interseção de diferentes lógicas terapêuticas esteve Elliott Joslin ${ }^{8}$, médico norte-americano reconhecido como o primeiro diabetologista do mundo, que enfatizou a educação dos diabéticos como fator crucial do tratamento. Embora tivesse sido o impulsionador de investigações no campo da dietética, nomeadamente as de Frederick Allen, não tinha uma postura radical, permitindo a ingestão de hidratos de carbono em pequenas quantidades. Membro do Comité para a aplicação da insulina formado em 1922, Joslin foi dos primeiros médicos nos EUA a experimentar a insulinoterapia em humanos com a primeira insulina comercial produzida pela indústria farmacêutica Eli Lilly (Cerqueira Vieira, 2020, p. 111).

Porém, o grande legado de Joslin concretizou-se no campo da educação dos diabéticos, tanto em doentes como em profissionais de saúde. A complexidade de que se revestiu a terapêutica da diabetes, após a descoberta da insulina, levou a uma domesticação dos cuidados médicos, tendo o doente um papel relevante no autodiagnóstico e tratamento na esfera doméstica. Como defende Gardner (2019, p. 175), a maior parte dos cuidados médicos tinham lugar no espaço doméstico, e a cozinha era identificada como o local onde se mediam e quantificavam ingredientes destinados à alimentação - usando uma tecnologia muito própria como as balanças, as colheres de chá, as colheres de sopa, as chávenas, etc. - se esterilizavam as seringas e as agulhas destinadas à administração de insulina, e ainda onde se realizavam análises às urinas. $\mathrm{O}$ lar dos diabéticos, e particularmente a cozinha, transformaram-se em laboratórios domésticos.

A preocupação com a educação dos diabéticos surgiu bem cedo quando Joslin iniciou a sua prática clínica e fundou em Boston a Joslin Clinic em 1898, vocacionada para o tratamento e educação dos diabéticos. Várias décadas mais tarde, em 1957, iria fundar a Diabetes Foundation, Inc., bem próximo do New England Deaconess Hospital, com um triplo objetivo: educar, cuidar, investigar (Martin, 1958, p. 390).

Primeiro, promover a educação dos diabéticos, ensinando-Ihes regras de vida, higiene, autoinjecção de insulina, recolha de urina, confeção de refeições, entre outras valências, já que a sua filosofia era "teaching is cheaper than nursing" (Barnett, 1998, p. 45). 
A educação era compreendida como um conceito e intervenções alargadas, já que os novos diabéticos eram ensinados pelos médicos, enfermeiros e incluso mantinham contactos com os diabéticos veteranos, que tinham um papel importante no reforço da adoção de estilos de vida saudáveis, ao mesmo tempo que eram um suporte psicológico relevante pela partilha de histórias de vida bem sucedidas (Martin, 1958, p. 391).

Em segundo lugar, a prestação de cuidados médicos e de enfermagem em regime de ambulatório, minorando os custos com os cuidados de saúde em hospitais ou clínicas gerais mais onerosas, bem como ter acesso a cuidados específicos pela especialização destas instituições (Thorn \& Watkins, 1982, p. 787).

O terceiro objetivo passava pela promoção da investigação diabetológica em múltiplas vertentes, desde a organização dos registos médicos, produção de estudos estatísticos e elaboração de protocolos de tratamento (Martin, 1958, p. 391; Barnett, 1998, p. 47).

Adepto do aprender fazendo, Joslin preconizava a adoção de novos métodos no tratamento ambulatório dos doentes, enfatizando a educação e os cuidados à cabeceira dos doentes, o que permitia reduzir os custos no tratamento hospitalar como também envolvê-los com seu o tratamento e condição (Martin, 1958, p. 390). A evolução do tratamento doméstico da diabetes exigiu uma maior circulação do conhecimento médico e a criação de uma linguagem comum que servisse tanto o clínico como o doente, o que na prática se consubstanciou na publicação de manuais que permitissem uma orientação imediata no tratamento. Como defende Gardner (2019, p. 178), os manuais permitiram estandardizar os cuidados aos doentes diabéticos, e Elliott Joslin foi um dos primeiros médicos a publicar um manual deste género.

Em 1918 foi publicada a primeira edição do «A diabetic manual for the mutual use of doctor and patient» com o objetivo de o médico ter uma ferramenta para educar os doentes e permitir um tratamento adequado sem necessidade de assistência médica direta, porque, segundo Joslin (1919, p. vii), "those who undestand the disease the best live the longest". Existia um objetivo claro de empoderamento do diabético, que era educado para conseguir tratar-se de acordo com a sua condição, tendo Joslin criado uma abordagem conhecida como Diabetes Self-Management Education, mais tarde implementada por Ernesto Roma em Portugal (Cerqueira Vieira, 2020, pp. 125-126). Apesar de nas duas primeiras edições - a de 1918 e 1919 - não existir menção à insulinoterapia, porque ainda não havia sido descoberta, a estrutura do livro foi mantida até 1960, quando foi editado pela última vez (Cerqueira Vieira, 2020, p. 127), o que mostra a pertinência e atualidade constante do manual ao longo de várias décadas.

\subsection{TRABALHO SOCIAL APLICADO À DIABETOLOGIA}

O espaço de atuação da medicina clínica no campo da diabetologia revelou-se insuficiente para lidar com uma doença crónica com várias e gravosas complicações associadas. No tratamento da diabetes seria por si só suficiente a perícia clínica? A educação dos doentes deveria realizar-se estritamente no espaço da consulta e dirigida pelo médico? Foram estas as questões colocadas nos inícios do século XX por alguns médicos norte-americanos, entre eles Richard Cabot.

Desde o exórdio da medicina social em meados do século XIX, a doença nos humanos não era vista apenas como um fenómeno biológico que ocorria na natureza. Pelo contrário, a doença humana era mediada e modificada pela atividade social e pelo ambiente cultural que essa atividade criava (Rosen, 1947, p. 674). Deste modo, a medicina clínica per se não seria capaz de debelar as doenças dependentes de causalidade social, designadamente as que dependiam dos contactos sociais, dos ambientes conspurcados, das práticas e costumes anti-higiénicos ou ainda do consumo alimentar exagerado ou deficitário, como nas doenças da nutrição.

Neste contexto há que destacar o trabalho pioneiro de Richard Cabot, na criação do serviço social em contexto hospitalar, para apoio aos doentes em ambulatório. Cabot acreditava que a medicina hospitalar se tinha divorciado do contexto social da doença, ao mesmo tempo que o trabalho médico e de enfermagem se realizava essencialmente nas instalações hospitalares, não havendo um seguimento dos doentes nos seus lares (O'Brien, 1985, p. 541). Por outro lado, acreditava na importância da profissionalização de vários prestadores de cuidados de saúde, nomeadamente das enfermeiras e das assistentes sociais, e num trabalho multidisciplinar e interdependente entre médico, assistente social e doentes.

Numa obra de grande importância médico-social publicada pela primeira vez em 1909 e com republicações sucessivas - "Social Service and the Art of Healing» - Richard Cabot defendia que vários problemas de saúde pública tinham origem nas condições de vida da população como a pobreza, a imoralidade, a ignorância, a superlotação das casas, as condições de trabalho, etc., defendendo um trabalho conjunto entre o médico e um grupo emergente de trabalhadores, os Trabalhadores Sociais ou Assistentes Sociais, que ti- 
nham um papel importante na deteção dos problemas sociais, como má nutrição, ambientes conspurcados, alcoolismo, acidentes laborais, doenças sociais como a tuberculose entre outros (Cabot, 1914, p. vii-viii).

Tecendo críticas aos médicos do seu tempo, Richard Cabot acusava os médicos de estarem interessados sobretudo nos casos clínicos (que ele considerava de natureza acidental) e não no plano de fundo das doenças preveníveis. Chamou à atenção para o papel determinante dos trabalhadores sociais na dinamização de grandes campanhas contra a tuberculose, contra o leite conspurcado, por uma lei alimentar ou pela criação de uma escola de Higiene nos EUA (Cabot, 1914, p. 36). Como referiu Mary Ellen Richmond (1917, p. 35), a pioneira do trabalho social nos EUA, Richard Cabot foi o mentor do serviço médico-social a partir de 1905 com a organização do primeiro departamento de serviço social num hospital norte-americano. A partir do Departamento de Ética Social, que presidia na Universidade de Harvard, passou a ensinar ética aplicada, que se focava na aplicação prática da ética junto das comunidades intervencionadas pelas assistentes sociais. Escorada em conceitos como virtude, responsabilidade e cuidado (Garchar \& Kaag, 2013, p. 556), a formação dos estudantes de medicina de Harvard passou a integrar trabalho médico-social no Massachussets General Hospital (Richmond, 1917, p. 36).

Na mesma época e na mesma cidade ocorreu também um avanço importante na formação das enfermeiras especialistas em diabetes, surgido no contexto do New England Deaconess Hospital School of Nursing, onde vários professores da Harvard Medical School, como Elliott Joslin, lecionavam. A partir de 1922, a Deaconess School of Nursing pôs em prática um programa rigoroso de formação de enfermeiras capazes de se engajarem com os doentes e suas famílias e providenciar uma educação diabetológica tão completa quanto possível na altura. Joslin chamou a esta tipologia de enfermeiras as wandering diabetic teaching nurse (Barnett, 1998, p. 43).

Num ambiente educativo estimulante, as enfermeiras aprendiam cuidados específicos no tratamento de diabéticos, insulinoterapia e técnica de injeção, manuseamento do vestuário do diabético, calorimetria, dietética e nutrição, educação dos diabéticos e suas famílias (Barnett, 1998: 43). Num espaço adjacente ao hospital foi construído um chalé - o William Nast Broadbeck Cottage - onde as enfermeiras tinham a possibilidade de pôr em prática os seus conhecimentos. Aí realizavam-se palestras e aulas para os diabéticos e suas famílias, bem como para enfermeiras e médi- cos, funcionando ainda uma cozinha e um restaurante, sendo as enfermeiras responsáveis pela preparação das refeições para os doentes e, mais tarde já em 1934, promoveram-se aulas sobre cozinha, nutrição, dietética e alimentos (Allen, 2003: 979 e 981).

No primeiro quartel do século $X X$ ficaram definidas as bases da diabetologia, escoradas em três pilares fundamentais: a insulinoterapia, a educação do diabético e o trabalho social. Foram em grande medida estes os conhecimentos que Ernesto Roma assimilou durante o período de estágio nos EUA e com eles veio a estabelecer a diabetologia social em Portugal.

\section{ORGANIZAÇÃO DA DIABETOLOGIA SOCIAL E PRIMEIRAS CONCRETIZAÇÕES EM PORTUGAL}

A organização da diabetologia social em Portugal correspondeu historicamente a um processo de simbiose entre a diabetologia, enquanto área de intervenção multidisciplinar, e o trabalho social, enquanto sustentáculo do processo de tratamento e de educação médica. Nos inícios do século XX, a diabetologia passou a ser uma nova área médica reconhecida internacionalmente, mas do domínio da Clínica Geral ou da Medicina Interna. Em Portugal, a diabetes era tratada sobretudo pelos Internistas, antes de ser anexada pela especialidade Endocrinologia-Nutrição, esta reconhecida somente pela Ordem dos Médicos em 1956 (Cerqueira Vieira, 2018, p. 41).

No que diz respeito à diabetologia social em Portugal, sabemos que o seu pioneiro e mentor foi o médico Ernesto Galeão Roma (1887-1978). Ensinando e exercendo clínica no Hospital de Santa Marta desde 1913, Ernesto Roma foi incitado por Carlos Bello de Morais, médico e professor na Faculdade de Medicina da Universidade de Lisboa, para se especializar em ética nos Estados Unidos da América com Richard Cabot. Mas quando chegou a Boston em dezembro de 1922, já com 35 anos de idade, o que viu e assistiu fizeram-no desistir dos projetos ligados à ética, para se dedicar à diabetologia, que acabara de nascer pela mão de Joslin Elliott (Veloso \& Correia, 2017, p. 248).

O ponto de viragem decisivo no tratamento da diabetes foi a descoberta e introdução da insulina, o que tinha ocorrido entre os finais de 1921 e os inícios de 1922, e consagrado mundialmente em 1923 com a atribuição do Prémio Nobel de Fisiologia ou Medicina aos responsáveis pela descoberta. O jovem médico Ernesto Roma começou o seu estágio em Boston, no epicentro de uma revolução terapêutica e assistiu ao desenvolvimento da diabetologia e do trabalho social, 
o que veio a influenciar decisivamente a sua carreira em Portugal.

Determinado a dedicar-se à diabetologia, Ernesto Roma regressou em outubro de 1923 e foi, presumivelmente, o primeiro médico no país a utilizar a insulina ${ }^{9}$, numa época em que ainda havia escassa informação sobre esta nova substância terapêutica tanto em Portugal como na Europa. A sua reintegração no Hospital de Santa Marta, em Lisboa, no serviço do Prof. Carlos Bello de Morais, coincidiu com o início da insulinoterapia em Portugal como o próprio revelou:

[...] voltei a Portugal, desligando-me do Prof. Cabot [...] aqui chegado, iniciei a prática do tratamento pela insulina, no serviço do Prof. Belo de Morais, com quem trabalhei muito tempo, que no meu regresso me recebeu muitíssimo bem, e me colocou logo a trabalhar no seu sector do Hospital de Santa Marta. Aí comecei então a fazer o tratamento da diabetes com o novo medicamento, prática que tornei extensiva ao consultório do Prof. Belo de Morais. E note que os doentes afluíam sem qualquer espécie de publicidade; eram eles próprios que faziam propaganda uns junto dos outros ${ }^{10}$.

Numa época em que os artigos publicados em periódicos médicos sobre diabetologia eram raros em Portugal, apareceram a partir de 1924 alguns artigos publicados na revista portuguesa "Lisboa Médica», inaugurada nesse ano e editada pelo Hospital de Santa Marta, o mesmo em que Ernesto Roma trabalhava. Logo em 1924 o médico e professor da Faculdade de Medicina de Lisboa, Pulido Valente publicou um conjunto de artigos intitulados "Lições sobre a diabetes»" (Valente, 1924), onde abordou os diversos aspetos diabetológicos, dedicando parte da quarta lição à insulina, fazendo um historial da sua descoberta e um resumo de experiências terapêuticas ensaiadas por investigadores como Frederick Allen, Frederick Banting, James Collip, entre outros ${ }^{11}$.

Em 1925, o médico e professor da Faculdade de Medicina da Universidade de Lisboa, Fernando da Fonseca $^{12}$, que trabalhava no mesmo Hospital de Santa Marta, publicou um artigo científico intitulado «Insulinas» (Fonseca, 1925), com a descrição de dois casos clínicos tratados nesse hospital com marcas diferentes de insulina, procurando Fernando da Fonseca fazer corresponder dosagens das diferentes marcas comercializadas ${ }^{13}$.

Como mencionaram Veloso \& Correia (2017, pp. 248-249), em nenhum dos artigos, o de Pulido Valente e o de Fernando da Fonseca, Ernesto Roma foi mencionado como precursor da diabetologia ou importador de uma nova terapêutica. No Hospital de Santa Marta corria o boato de que aquando da chegada de Ernesto Roma a Portugal, este ter-se-ia reunido com Pulido Valente aconselhando-o a preparar uma série de aulas sobre a diabetes, que mais tarde seriam publicadas e o que veio a acontecer em 1924 . Todavia, no conjunto de artigos que publicou, em momento algum Pulido Valente fez menção a Ernesto Roma (Veloso \& Correia, 2017, pp. 248-249).

A primeira comunicação e publicação conhecida de Ernesto Roma sobre o tratamento da diabetes pela insulina só veio a acontecer entre 1925-1926. Aquando da comemoração do centenário da criação da Régia Escola de Cirurgia de Lisboa, Ernesto Roma, então Chefe de Clínica do Hospital de Santa Marta, foi convidado a dar uma conferência, tendo escolhido como tema o tratamento do coma diabético, conferência essa que foi publicada em 1926.

Veloso \& Correia (2017, p. 249) defendem que no início Ernesto Roma não tirou nenhum proveito pessoal dos conhecimentos adquiridos em diabetologia e insulinoterapia que aprendera nos EUA. De facto, o regresso de Ernesto Roma fez-se de forma subtil, voltando a exercer clínica hospitalar e privada associado, ou sob tutela, ao já referido Carlos Bello de Moraes.

O seu papel na diabetologia portuguesa vincou-se por outra via que não a hospitalar e fazendo recurso aos conceitos e conhecimentos que aprendeu e transferiu para a realidade portuguesa. Não hesitando em designar a insulina de "uma das mais maravilhosas descobertas da Humanidade" (Roma, 1926, p. 78), a sua grande preocupação era, porém, pedagógica e filantrópica. A estada de Roma nos EUA permitiu-lhe ter contacto direto com os métodos usados no trabalho social com os doentes e na educação dos diabéticos que aprendera diretamente com Cabot e Joslin. O seu regresso a Portugal e os primeiros anos de trabalho, já na era da insulina, conduziram-no a outros tipos de preocupações - por um lado a do problema da educação dos diabéticos, para consolidação do tratamento ambulatório que a insulinoterapia havia permitido; por outro lado o problema dos diabéticos pobres sem meios para adquirir a insulina.

Estas realizações só vieram a ser possíveis a partir de 1926, aquando da fundação da Associação Portuguesa dos Diabéticos Pobres (APDP), que foi muito mais do que uma instituição de assistência. Na prática a APDP funcionou como a incubadora da diabetologia social em Portugal, permitindo a Ernesto Roma pôr em prática os princípios do tratamento moderno da dia- 
betes - insulinoterapia e educação dos diabéticos - e promover o trabalho social que aprendera com Cabot.

\subsection{AÇÃo ASSISTENCIAL E EDUCATIVA DA AsSOCIAÇÃo PORTUguesa dos Diabéticos POBRES (APDP)}

Na génese da fundação da APDP esteve a constatação, por parte de Ernesto Roma, de que existia um número significativo de diabéticos incapazes de suportarem financeiramente o seu tratamento, e por isso impedidos de acederem a uma educação conveniente neste campo ${ }^{14}$. Teve a ideia de sensibilizar os pacientes que acorriam ao seu consultório para a questão e pediu-lhes apoio e ajuda para a fundação de uma associação beneficente que pudesse atender aos pacientes economicamente desfavorecidos, assumindo-se esta como:

"uma instituição humanitária e de beneficência, que tem por fim proteger e socorrer, a dentro da sua esfera de acção, os doentes diabéticos, de qualquer sexo, edade e condição, reconhecidamente pobres e como tal careçam de auxílio extranho, quer sejam ou não sócios d'esta Instituição, e combater por todas as maneiras possíveis a diabétes e as suas consequências" ${ }^{15}$.

Como mencionamos a questão da insulinoterapia era premente. Desde logo a APDP se propôs a fornecer aos diabéticos pobres a insulina que precisavam e outros produtos, alimentares ou não, para um tratamento mais eficaz da doença ${ }^{16}$. Iniciou a sua atividade em 1927 dando assistência a 14 doentes, com os quais despendeu 20.000 unidades de insulina nesse ano, mas o número de diabéticos assistidos aumentou anualmente. A análise do Quadro 1 permite-nos perceber que nos primeiros cinco anos de atividade, a assistência aos doentes diabéticos passou de apenas 14 para 410 , quase trinta vezes mais o seu número. Houve um aumento significativo do número de diabéticos assistidos a partir de 1929-1930, que se ficou a dever ao facto de os Hospitais Civis de Lisboa canalizarem os diabéticos pobres seguidos em ambulatório para os serviços clínicos da APDP ${ }^{17}$. O número de assistidos pelas filiais do Porto e Coimbra era residual - dez doentes em cada filial - pelo que o aumento se verificou sobretudo na sede em Lisboa.

Consequência do aumento de assistidos foi o aumento de unidades de insulina utilizadas na terapêutica, passando em cinco anos de 20 mil unidades para 922 mil unidades (ver Quadro 1), o que exigia uma despesa avultada da APDP com esta substância e o recurso a mecenas e protocolos para poder suportar as despesas crescentes com este fármaco.
Quadro 1. Evolução do n. de doentes assistidos pela APDP nos primeiros cinco anos de atividade. Fonte: Relatórios e Contas da APDP de 1927 a 1932.

\begin{tabular}{|l|l|l|}
\hline Anos & $\begin{array}{l}\text { N.o de doentes } \\
\text { assistidos }\end{array}$ & $\begin{array}{l}\text { Unidades de } \\
\text { insulina utilizadas }\end{array}$ \\
\hline $1927-1928$ & 14 & 20.000 \\
\hline $1928-1929$ & 85 & 230.000 \\
\hline $1929-1930$ & 204 & 640.000 \\
\hline $1930-1931$ & 274 & 720.900 \\
\hline $1931-1932$ & 410 & 922.000 \\
\hline
\end{tabular}

Os custos com a insulina foram mitigados pela ajuda de algumas casas farmacêuticas sediadas em Lisboa, que vendiam a insulina a um preço especial à associação, designadamente o Instituto Pasteur de Lisboa, que comercializava a insulina americana da marca Lilly, e a Coll \& Taylor Lda., que comercializava a marca inglesa A.B. ${ }^{18}$. Com o objetivo de ajudar no combate à diabetes, também a Santa Casa da Misericórdia de Lisboa decidiu na sessão de 31 de outubro de 1927 estabelecer um subsídio mensal de 5 mil unidades de insulina à APDP ${ }^{19}$, aumentando a partir de outubro do ano subsequente o donativo para 10 mil unidades por mês ${ }^{20}$.

A ajuda à APDP fazia-se ainda pela filiação à associação, na qualidade de sócio contribuinte ou sócio protetor (instituições), com o pagamento de uma quota mensal, mas igualmente com os donativos ocasionais de pessoas e instituições interessadas e solidárias com a causa da luta contra a diabetes. Para além de donativos particulares, a APDP recebeu subsídios da Direção Geral da Assistência, do Governo Civil de Distrito de Lisboa, da Manutenção Militar, da Companhia Portuguesa de Higiene, entre outros ${ }^{21}$. A par das insulinas houve auxílio das instituições referidas na oferta de pão de glúten, águas minerais, seringas, agulhas, licor de Fehling para o exame às urinas, balanças para pesagem dos alimentos.

Outro âmbito de atuação da APDP foi na área da educação dos diabéticos. Seguindo os preceitos aprendidos em Boston, Ernesto Roma pôs em prática um modelo de educação do diabético que aprendera em Boston. Ao que se sabe Ernesto Roma já dava palestras aos seus doentes da clínica privada desde que regressara dos EUA ${ }^{22}$. Porém, só a partir de 1930-1931 aparecem documentadas nos «Relatórios e Contas» as palestras de ensinamento dietético e profilático.

As palestras, designadas de "consulta de educação médica e dietética", realizavam-se às quartas-feiras às 
18 h30 e tinham a duração de uma hora, sendo os lugares ocupados mediante um sistema de senhas distribuídas antes da consulta. Entre os temas destacavam-se os princípios gerais da alimentação, noções básicas sobre calorias, hidratos de carbono, proteínas e gorduras, localização do órgão responsável pela doença, principais complicações e prevenção, coma diabético e sintomas, sopas para diabéticos, pratos principais e pratos do meio, etc. ${ }^{23}$.

Em 1931 na qualidade de Diretor Clínico da APDP, Ernesto Roma publicou um compêndio intitulado "Conselhos aos diabéticos», com a pretensão de educar e esclarecer com informações de base científica os diabéticos e seus cuidadores. Os conselhos permitiriam adotar o melhor plano de tratamento, saber como variar a dieta sem comprometer o estado de saúde, difundir os cuidados higiénicos para esta tipologia de doentes. Na prática o "Conselhos aos diabéticos» (Roma, 1931) funcionou como um auxílio para a boa educação dos diabéticos e mesmo profissionais de saúde, à semelhança do que Elliott Joslin fez com a sua obra "A diabetic manual for the mutual use of doctor and patient» (Joslin, 1919).

Mas, contrariamente à extensão da obra de Joslin, os "Conselhos aos diabéticos» de Ernesto Roma era um compêndio de 20 páginas, e mais 8 páginas de anexos, onde o autor enfatizava sobretudo a questão da dietoterapia. Houve um enorme cuidado na explicação da composição nutricional dos alimentos, na quantidade em peso de alimentos que podia ser inserida em cada refeição pelo diabético, na composição de dietas alimentares por cada dia da semana, medidas de higiene, interpretação dos sinais de coma diabético e precauções a tomar.

Em 1931 foi inaugurada uma publicação, que pretendia ser periódica, o «Boletim da Associação Protectora dos Diabéticos Pobres», de distribuição gratuita e com o objetivo de propagandear as ações e obra da APDP, veicular notícias, noticiar avisos e educar os leitores diabéticos (Cerqueira Vieira, 2020, p. 154). As dificuldades da APDP nessa época tornaram a publicação inconstante. Entre 1931 e 1958 foram apenas publicados onze números do boletim, o que mostra que esta era uma publicação irregular e descontinuada no tempo (Cerqueira Vieira, 2020, p. 155), significando que apesar da boa intenção na divulgação de preceitos terapêuticos e higiénicos o seu alcance foi reduzido pela exiguidade de números publicados, não se podendo considerar como um meio educativo de pleno direito, como foram outras publicações médico-sociais da época ${ }^{24}$.
Outra vertente muito vincada desta associação esteve ligada ao trabalho social que desempenhou. Para além do tratamento atualizado, da educação esmerada e do acompanhamento zeloso dos diabéticos, a APDP oferecia apoio em vários domínios, desde a instrução dos doentes pobres de acordo com o programa de palestras científico-dietéticas, ao ensino do método de análise da glicose nas urinas, à farmácia social, até aos protocolos mantidos com diversas instituições.

A APDP criou nos primeiros anos da sua existência um laboratório destinado à realização de exames clínicos e bromatológicos, o que resolveu a questão da gratuitidade das análises aos diabéticos pobres ${ }^{25}$. Os diabéticos pobres contavam com serviços de análises clínicas gratuitas, serviços esses que estavam a cargo dos médicos António Morais David e Carlos Trincão, respetivamente Assistente e Chefe do Laboratório de Clínica Médica da Faculdade de Medicina de Lisboa. 0 exame bromatológico à composição química dos alimentos era assegurado pelo Capitão José Pedro Alves, que era à época farmacêutico-químico e chefe do Laboratório da Manutenção Militar ${ }^{26}$.

Influenciado pelas ideias de profissionalismo dos trabalhadores sociais defendidas por Richard Cabot, Ernesto Roma pugnou pela formação de todos os funcionários a exercer funções na APDP, que incluía tantos os médicos como as rececionistas, que eram sujeitos a ações de formação técnica, tornando-os capazes de assegurar na sua área de atuação os melhores cuidados e atenção aos doentes. Fazia parte da formação dos funcionários, por exemplo, a assistência às palestras científico-dietéticas. Na década de 1940 foram contratados os serviços de uma enfermeira norte-americana, Sara Norton, que se dedicava exclusivamente à formação e atualização do pessoal de enfermagem e também de uma podologista alemã, Hilda Greenfontein, se responsabilizou pela formação dos médicos e dos enfermeiros nos cuidados e tratamento do pé diabético (Correia \& Boavida, 2006, p. 45).

Houve ainda o cuidado de estabelecer um protocolo que beneficiasse os doentes carenciados. Em 19291930, a APDP acordou com os Hospitais Civis de Lisboa que receberia na sede os diabéticos em regime ambulatório encaminhados pelo hospital e em contrapartida os doentes assistidos pela APDP poderiam realizar exames no serviço de radiologia de forma gratuita ${ }^{27}$. Visto que nos início a APDP não dispunha de instalações de tipo hospitalar que permitisse o internamento dos doentes, no ano de 1930, a APDP estabeleceu um acordo com a Direção do Hospital da Ordem Terceira de S. Francisco, em Lisboa, permitindo aos diabéticos 
assistidos pela associação poderem beneficiar de hospitalização especializada gratuita nos hospitais pertencentes à Ordem Terceira de São Francisco, sendo também extensível aos diabéticos inscritos nas filiais da APDP $^{28}$. Nos anos seguintes o acordo passou a incluir o acesso à consulta externa e ao serviço de radiologia ${ }^{29}$.

\section{CONCLUSÃO}

A génese da diabetologia social em Portugal, que institucionalmente foi promovida e desenvolvida no seio da APDP, não ocorreu antes de 1926. Nem a existência de estudos científicos nos meios académicos nem a introdução da insulina em Portugal no tratamento da diabetes foram suficientes per se para a criação da diabetologia social. Foi em larga medida o movimento associativista empreendido por Ernesto Roma em Lisboa que permitiu o desenvolvimento desta área em Portugal.

Foi o trabalho no terreno de especialistas de renome mundial como Elliott Joslin e Richard Cabot, com quem Ernesto Roma estagiou e aprendeu, que provaram ser insuficiente a terapêutica convencional perante uma doença crónica e incurável. Face à diabetes era necessário que o paciente aprendesse a viver de acordo com a sua condição e isso exigia uma educação em "banda larga". A descoberta da insulina permitiu dar autonomia ao doente diabético e tratá-lo em ambulatório, mas para tal era necessário ensinar técnicas de autoinjecção e análise à urina, enquanto se ensinava a comer segundo uma aritmética própria e estrita e, de igual modo, se ensinava a interpretar os sintomas da doença.

Em 1926 foi fundada a APDP, a primeira deste género no mundo ${ }^{30}$, com o propósito de desenvolver a diabetologia social. Em poucos anos, a APDP tornou-se um centro de estudos da diabetes e do doente diabético, tendo contribuído para a formação de vários médicos de Clínica Geral, Medicina Interna e Endocrinologia, enfermagem especializada e podologia; um centro de diagnóstico precoce através dos seus programas de sensibilização e rastreamento da diabetes; um centro de estabilização do doente com a finalidade de reabilitar o diabético tanto do ponto de vista clínico como social; e um centro educacional em múltiplas frentes, da insulinoterapia à dietoterapia.

Ernesto Roma é considerado o precursor na introdução da dietética e nutrição como ciências auxiliares da medicina e da diabetologia ${ }^{31}$, mas igualmente precursor na formação das primeiras assistentes sociais em Portugal. Além do projeto de formação de trabaIhadores sociais dentro da APDP, foi igualmente professor dos cursos de ensaio de Serviço Social realizados em Portugal em 1929-1930, que passaram a realizar-se regularmente entre 1935 e 1939, mas somente aprovados legalmente em 1939 pelo Decreto-Lei n.o 30135, de 14 de dezembro de 1939, realizados à data no Posto de Protecção à Infância de Lisboa. Tal como Richard Cabot havia sido precursor destes cursos nos EUA, Ernesto Roma foi dos primeiros a colaborar na formação das profissionais de trabalho social em Portugal.

\section{NOTAS AL FINAL}

1 Diabetologia social é um conceito derivado da medicina social. Esta surgiu historicamente ligada à necessidade de dar resposta a doenças criadas pelo industrialismo, como forma de aliar a medicina clínica e preventiva (Galdston, 1951). Neste sentido a diabetologia social tem uma vocação educadora, pretendendo, mais do que administrar a terapêutica específica, educar e empoderar o doente ensinando-Ihe a higiene, a técnica de injeção de insulina, a dieta adequada, o cálculo de valores nutricionais, o autoexame à urina, etc. (Lisboa, 1976).

2 Veja-se os seguintes estudos: Bankston, John (2002), Frederick Banting and the discovery of insulin, Bear, Mitchell Lane Publisher; Bliss, Michael (2017), The Discovery of Insulin, Toronto, University of Toronto Press; Engelhardt, Dietrich (ed.) (1989), Diabetes Its Medical and Cultural History: Outlines - Texts - Bibliography, Berlim, Springer-Verlag; Hurley, Dan (2010), Diabetes Rising: How a modern disease became a modern pandemic, and what to do about it, New York, Kaplan Publishing; lonescu-Tirgoviste, Constantin, \& Buda, Octavian (2017), "Nicolae Constantin Paulescu: The first explicit description of

the internal secretion of the pancreas", Acta medico-historica Adriatica, 15(2), pp. 303-322; Mazur, Allan (2011), "Why were 'starvation diets' promoted for diabetes in the pre-insulin period?" Nutrition Journal, 10(23), pp. 1-9; Stylianou, Charilaos, \& Kelnar, Christopher (2009), "The introduction of successful treatment of diabetes mellitus with insulin", Journal of the Royal Society of Medicine, 102(7), pp. 298-303; Tattersall, Robert (2009), Diabetes: The Biography, Oxford, Oxford University Press; Westman, Eric C., Yancy, William S., \& Humphreys, Margaret (2006), "Dietary treatment of diabetes mellitus in the pre-insulin era (1914-1922)", Perspectives in Biology and Medicine, 49(1), pp. 77-83.

3 Para o século XIX estão identificadas oito teses inaugurais das Escolas Médico-cirúrgicas de Lisboa e do Porto: Simões, José (1863), Observação de um caso de diabetes seguido de algumas considerações sobre a symptomologia, a anatomia pathologica e o tratamento (Tese Inaugural), Lisboa, Escola médico-cirúrgica de Lisboa [manuscrita]; Silva, Manuel (1868), Alguns phenómenos da diabete (Tese Inaugural), Lisboa, Typographia Universal de Thomaz Quintino Antunes; Centeno, Dominguez (1888), Contribuição para o estudo do coma diabético (Tese 
Inaugural), Lisboa, Typographia e Lithographia da Papelaria Progresso; Maia, Aires de Araújo (1873), Qual a origem da glycose na diabéte saccharina? (Tese Inaugural), Porto, Imprensa Popular de Mattos Carvalho \& Vieira Paiva; Melo, André (1882), A Diabete Assucarada: seu esboço etiológico, pathogenico e therapeutico (Tese Inaugural), Porto, Typographia Occidental; Sousa, Joaquim (1892), Estudo sobre a pathogenia da diabete assucarada (Tese Inaugural), Porto, Typographia Occidental; Oliveira, Lucindo (1895), Breve estudo sobre a pathogenia da diabete (Tese Inaugural), Porto, Papelaria e Typographia de Manuel J. Alves d'Azevedo; Baptista, Alberto (1898), Breves considerações sobre a pathogenia da diabete (Tese Inaugural), Porto, Typographia Pereira.

4 Veja-se o importante estudo de Allen «Studies Concerning Glycosuria and Diabetes» (1913) e "Total dietary regulation in the treatment of diabetes» (1919), este último em coautoria com Edgar Stillman e Reginald Fitz.

5 «Os 50 anos da Associação Protectora dos Diabéticos de Portugal: Entrevista com o Dr. Ernesto Roma», Notícias Médicas, n.o 354, 27/04/1976.

6 Veja-se a propósito do estado sanitário em Portugal no primeiro quartel do século XX o trabalho de Cerqueira Vieira (2019).

7 Veja-se sobre este tema Han (2014).

8 Reconhecido como o primeiro diabetologista do mundo, Elliott P. Joslin destacou-se pelos cargos e filiações que seguidamente sintetizamos: Tenente-Coronel do Exército Norte-Americano, professor da Harvard Medical School, consultor do Boston City Hospital, Médico-em-Chefe Honorário do New England Deaconess Hospital, fundador da Diabetes Foundation, Inc., Diretor do Nutrition Laboratory da Carnegie Institution, membro da Academia Americana de Artes e Ciência, da Associação dos Médicos Americanos, Sociedade Americana de Filosofia, grau de Doutor Honoris Causa em Ciência pela Universidade de Harvard e pela Universidade de Toronto, Doutor Honoris Causa em Medicina pela Universidade de Düsseldorf e a Medalha Banting da Associação Americana de Diabetes e ainda os títulos honorários franceses de Membro da Academia Francesa e da Academia das Ciências, Professor do Collège de France e Comendador da Légion d'Honneur (Root, 1962: 38-39).

9 Ao nível europeu, presumivelmente o primeiro médico a fazer uso da insulina foi o espanhol Rosendo Carrasco y Formiguera, professor agregado de Nutrição na Universidade Autónoma de Barcelona, que em 1921-1922 também trabalhou na Harvard Medical School com Walter B. Cannon em temas de endocrinologia e diabetologia publicando trabalhos importantes nesse domínio. Regressou a Espanha em 1922 com conhecimentos de insulinoterapia (Orozco Acuaviva, 1999, p. 169-170).

10 «Os 50 anos da Associação Protectora dos Diabéticos de Portugal: Entrevista com o Dr. Ernesto Roma», Notícias Médicas, n.o 354, 27/04/1976.

11 Recorde-se que Pulido Valente foi um dos médicos e clínicos mais relevantes da sua geração em Portugal, sobretudo no domínio da medicina interna. Foi igualmente um intelectual português que se empenhou, para além da clínica médica e da investigação científica, notabilizou-se no ensino da medicina e teve uma posição política pública de oposição ao regime político encabeçado por António de Oliveira Salazar vulgarmente conhecido como Estado Novo (Veloso, 2017, p. 199; In Memoriam, 1989).

12 Tal como Pulido Valente, também Fernando da Fonseca foi um prestigiado professor de Doenças Infetocontagiosas e de Pro- pedêutica Médica e também tinha fortes convicções políticas de oposição ao Estado Novo (Botelho, 1995).

13 Em 1925 utilizava-se na terapêutica da diabetes, no Hospital Escolar de Santa Marta, onde funcionava a Faculdade de Medicina de Lisboa, as marcas de insulina Merck, Léo, Byla, Toronto e Lilly (Cf. Fonseca, 1925).

14 «Os 50 anos da Associação Protectora dos Diabéticos de Portugal: Entrevista com o Dr. Ernesto Roma», Notícias Médicas, n. $354,27 / 04 / 1976$.

15 APDP (1927), Estatutos da Associação Protétora dos Diabéticos Pobres, Lisboa: Imprensa Lucas \& C. $\underline{\text {, }}$ p. 1.

16 APDP (1927), Estatutos da Associação Protétora dos Diabéticos Pobres, Lisboa: Imprensa Lucas \& C. a , p. 2.

17 APDP (1930), Relatório e Contas da gerência do ano económico 1929-1930, Lisboa, APDP, p. 3.

18 APDP (1929), Relatório e Contas da gerência do ano económico 1928-1929, Lisboa, Imprensa da Armada, p. 7.

19 AHSCML, Livro de Actas do Conselho de Administração da Misericórdia de Lisboa, Acta da sessão de 31 de outubro de 1927, p. 574.

20 APDP (1929), Relatório e Contas da gerência do ano económico 1928-1929, Lisboa, Imprensa da Armada, p. 21.

21 APDP (1930), Relatório e Contas da gerência do ano económico 1929-1930, Lisboa, APDP, p. 7-8.

22 «Os 50 anos da Associação Protectora dos Diabéticos de Portugal: Entrevista com o Dr. Ernesto Roma», Notícias Médicas, n.으 354, 27/04/1976.

23 "Serviços Clínicos e Dietéticos», Boletim da Associação Protectora dos Diabéticos Pobres, vol. 1, n.ㅇ 2, outubro de 1931.

24 Deve sublinhar-se que o funcionamento da APDP terá de ser visto no âmbito da política assistencial do Estado Novo. Será oportuno o seu enquadramento e relacionamento com outras instituições e sua valoração pelo próprio Estado, o que pode constituir outro artigo resultante da investigação em curso. Sobre a política de saúde do Estado Novo vejam-se, por exemplo, Andreia S. Almeida (2018), A Saúde no Estado Novo de Salazar: O nascimento do Ministério da Saúde e Assistência, Coimbra, Almedina; Daniela Cristina da Costa Ribeiro (2018). Políticas de Saúde no Período do Estado Novo (1933-1974), (Tese de Mestrado), Porto, Faculdade de Letras da Universidade do Porto. Para um maior enquadramento dos serviços e das instituições de saúde pode ser pertinente a consulta da obra de F. A. Gonçalves Ferreira (1990). História da saúde e dos serviços de saúde em Portugal, Lisboa, Fundação Calouste Gulbenkian.

25 APDP (1929), Relatório e Contas da gerência do ano económico 1928-1929, Lisboa, Imprensa da Armada, p. 6.

26 "Serviços Clínicos e Dietéticos», Boletim da Associação Protectora dos Diabéticos Pobres, vol. 1, n.ㅇ 2, outubro de 1931.

27 APDP (1930), Relatório e Contas da gerência do ano económico 1929-1930, Lisboa, APDP, p. 8.

28 APDP (1931), Relatório e Contas da gerência do ano económico 1930-1931, Lisboa, Indústrias Gráficas, p. 4.

29 APDP (1932), Relatório e Contas da gerência do ano económico 1931-1932, Porto, Tip. do Instituto Surdos-Mudos, p. 4

30 "Obituary Dr Ernesto Roma», IDF Bulletin, vol. 23, n.o 3, outubro de 1978, p. 7.

31 "Obituary Dr Ernesto Roma», IDF Bulletin, vol. 23, n.o 3, outubro de 1978, p. 7. 


\section{BIBLIOGRAFÍA}

Allen, Frederick M. (1913), Studies Concerning Glycosuria and Diabetes, Cambridge, Harvard University Press.

Allen, Frederick Madison; Stillman, Edgar; Fitz, Reginald (1919), Total dietary regulation in the treatment of diabetes, Nova Iorque, The Rockefeller Institute for Medical Research.

Allen, Nancy A. (2003), "The history of diabetes nursing, 19141936", The Diabetes Educator, 29 (6), pp. 976-989.

Almeida, Andreia S. (2018), A Saúde no Estado Novo de Salazar: O nascimento do Ministério da Saúde e Assistência, Coimbra, Almedina.

Barnett, Donald (1998), Elliott P. Joslin, MD: A Centennial Portrait, Boston, Joslin Diabetes Center.

Botelho, Luís S. (1988), "Contribuição para a história da endocrinologia em Portugal", Acta Médica Portuguesa, 1(2), pp. 181-185

Botelho, Luís S. (1995), “Figuras da medicina: Dr. Fernando da Fonseca", Medicina Interna, 2(3), pp. 202-203.

Bynum, William (2012), A Little History of Science, New Haven, Yale University Press.

Cabot, Richard C. (1914), Social Service and the Art of Healing, Nova lorque, Moffat, Yard and Company.

Cerqueira Vieira, Ismael (2019), “Aspetos do estado sanitário em Portugal no primeiro quartel do século XX". In: Silva, Helena da; Pereira, Rui M.; Bandeira, Filomena (eds.), Centenário da Gripe Pneumónica: A pandemia em retrospetiva, Portugal 1918/1919. Lisboa, IGAS/IHC/Comissão Nacional para a celebração do Centenário da Pneumónica, pp. 37-54.

Cerqueira Vieira, Ismael (2018). História da Endocrinologia em Portugal no Século XX. Coimbra: Sociedade Portuguesa de Endocrinologia, Diabetes e Metabolismo.

Cerqueira Vieira, Ismael (2020), A Diabetologia em Portugal: Contributos para a sua História, Lisboa, Sociedade Portuguesa de Endocrinologia, Diabetes e Metabolismo.

Coimbra, Elísio (1919), A diabetes pancreática: generalidades sobre diabetes (Tese de Doutoramento), Porto, Escola Tipográfica da Oficina de S. José

Correia, Luís; Boavida, José, (2006), Fotobiografia de Ernesto Roma. Lisboa: Associação Portuguesa dos Diabéticos de Portugal.

Costa, Celestino da (1952), "Comentários sobre o advento e a evolução da endocrinologia", Acta Endocrinologica Iberica, 2(2) pp. 90-116.

Costa, José (1922), Contribuição para o estudo do tratamento da diabetes açucarada pelo método de Allen, (Tese de Doutoramento), Lisboa, Faculdade de Medicina da Universidade de Lisboa.

Ferreira, Gonçalves (1990), História da saúde e dos serviços de saúde em Portugal, Lisboa, Fundação Calouste Gulbenkian, 1990.

Fonseca, Fernando da (1925), "Insulinas", Lisboa Médica, 2(4), pp. 177-183.
Galdston, lago (1951), "Social medicine and the epidemic constitution", Bulletin of the History of Medicine, 25 (1), pp. 8-21.

Garchar, Kimberly; Kaag, John (2013), “Classical American Philosophy and Modern Medical Ethics: The Case of Richard Cabot", Transactions of the Charles S. Peirce Society, 49 (4), pp. 553-574.

Gardner, Kirsten E. (2019), “'The Art of Insuline Treatment:' Diabetes, Insulin and the 1920s", Journal of Medical Humanities, 40 (2), pp. 171-180, [em linha], https://doi.org/10.1007/ s10912-017-9493-x

Graça, Manoel (1806), Tratado da Diabetes, Lisboa, Tipografia Lacerdina.

Han, Byung-Chul (2014), A Sociedade do Cansaço, Lisboa, Relógio D’Água.

Holmes, F. (1999), " La physiologie et la médicine expérimentale ». In : Grmek, M. (ed.), Histoire de la pensée médicale en Occident Vol. 3, Paris, Seuil, pp. 59-96.

Houssay, B. (1989), "The discovery of pancreatic diabetes: the role of Oscar Minkowski", In: Engelhardt, D. (ed.), Diabetes Its Medical and Cultural History: Outlines - Texts - Bibliography, Berlim, Springer-Verlag, pp. 350-357.

Jordão, Abel (1857), Considérations sur un cas de diabète (Tese de Doutoramento), Imprimerie de L. Martinet, Paris \{em linha], disponível em https://archive.org/details/BIUSante_TPAR1857x113 [consultado em 10/3/2020].

Jordão, Abel (1863a), "Diabete: Causas da diabete", Jornal da Sociedade das Sciencias Medicas de Lisboa, 27(3), pp. 81-82.

Jordão, Abel (1863b), "Diabete: Sobre algumas causas da diabete", Jornal da Sociedade das Sciencias Medicas de Lisboa, 27(2), pp. 41-44.

Jordão, Abel (1866), Sobre alguns symptomas da diabete: lição clínica feita na Escola de Medicina de Lisboa, Lisboa, Typographia da Gazeta de Portugal.

Joslin, Elliott P. (1919), A diabetic manual for the mutual use of doctor and patient, Filadélfia/Nova lorque, Lea \& Febiger.

Lain-Entralgo, Pedro (1978), Historia de la Medicina, Barcelona, Salvat.

Lisboa, Pedro (1976), “O Ensino e a prática da diabetologia clínica em Portugal", Boletim da associação Protectora dos Diabéticos de Portugal, 86-87-88, pp. 25-31.

Lisboa, Pedro (2002), “História da Diabetes I: da 'pré-história' à 'idade moderna'”. In: Duarte, Rui (ed.), Diabetologia Clínica, 3. $\underline{a}$ ed., Lisboa: Lidel, pp. 1-16.

Martin, Marguerite M. (1958), "A Teaching Center for Diabetics", The American Journal of Nursing, 58 (3), pp. 390-391.

O'Brien, Laurie (1985), "A Bold Plunge into the Sea of Values": The Career of Dr. Richard Cabot", The New England Quarterly, 58 (4), pp. 533-553.

Orozco Acuaviva, António (1999), Historia de la Endocrinología Española, Madrid, Ediciones Díaz de Santos.

Ribeiro, Daniela (2018). Políticas de Saúde no Período do Estado Novo (1933-1974), (Tese de Mestrado) Porto, Faculdade de Letras da Universidade do Porto. 
Richmond, Mary E. (1917), Social Diagnosis, Nova lorque, Russel Sage Foundation.

Roma, Ernesto (1926), "Tratamento do côma diabético", Lisboa Médica, 3(3), pp. 59-86.

Roma, Ernesto (1931), Conselhos aos diabéticos, Lisboa, Imprensa Nacional de Publicidade.

Root, Howard F. (1962), “Elliott Proctor Joslin (1869-1962)”, Harvard Medical Alumni Bulletin, 36 (3), pp. 38-39.

Rosen, George (1947), "What is Social Medicine? A Genetic Analysis of the Concept", Bulletin of the History of Medicine, 21 (5), pp. 674-733.

Tattersall, Robert (2009), Diabetes: The Biography, Oxford, Oxford University Press.
Thorn, P. A.; Watkins, Peter J. (1982), "Organisation of Diabetes Care", British Medical Journal (Clinical Research Edition), 285 (6344), pp. 787-789.

Valente, Pulido (1924), "Lições sobre a diabetes", Lisboa Médica, 1(4), pp. 224-239; 1(5), pp. 265-283; 1(6), pp. 329-339.

Veloso, A.J.; Correia, Luís (2017), "Ernesto Roma e a Associação dos Diabéticos Pobres". In: Veloso, A.J.; Mora, Luiz Damas; Leitão, Henrique (eds.), Médicos e Sociedade: Para uma História da Medicina em Portugal no século XX, Lisboa, By the Book, pp. 245-259.

Veloso, Barros (2017), "Pulido Valente e o triunfo da medicina anátomo-clínica". In: Veloso, António Barros; Mora, Luiz Damas; Leitão, Henrique (eds.), Médicos e Sociedade: Para uma História da Medicina em Portugal no século XX, Lisboa, By the Book, pp. 199-212. 